\title{
THE CONJECTURES ON CONFORMAL TRANSFORMATIONS OF RIEMANNIAN MANIFOLDS
}

\author{
MORIO OBATA
}

\section{Introduction}

Let $(M, g)$ be a Riemannian $n$-manifold with Riemannian metric $g$. Throughout this paper manifolds under consideration are always assumed to be connected and smooth.

For a smooth function $\rho$ on $M$, a Riemannian metric $e^{2 \rho} g$ is said to be conformally related, or conformal, to $g$. Let $h$ be a smooth map of $(M, g)$ into another Riemannian manifold $\left(M^{\prime}, g^{\prime}\right)$. If the Riemannian metric $h^{*} g^{\prime}$ induced on $M$ by $h$ is conformal to $g$, then $h$ is called a conformal map of $(M, g)$ into $\left(M^{\prime}, g^{\prime}\right)$. It is well-known that $h$ is conformal if and only if it preserves the angle between any two tangent vectors. $h$ remains conformal under any conformal changes of Riemannian metrics on $M$ and $M^{\prime}$ as well. If $h$ is a conformal diffeomorphism of $(M, g)$ onto $\left(M^{\prime}, g^{\prime}\right)$, then it is called briefly a conformorphism of $(M, g)$ onto $\left(M^{\prime}, g^{\prime}\right)$, and $(M, g)$ is said to be conformorphic to $\left(M^{\prime}, g^{\prime}\right)$ Via $h$. If furthermore $(M, g)=\left(M^{\prime}, g^{\prime}\right)$, then $h$ is called a conformal transformation or a conformorphism of $(M, g)$.

It is known that the group $C(M, g)$ of all conformorphisms of $(M, g)$ is a Lie group with respect to the compact-open topology. Let $C_{0}(M, g)$ denote the connected component of the identity of $C(M, g)$. If $g$ and $\bar{g}$ are conformal to each other, then $C(M, g)=C(M, \bar{g})$. The group $I(M, g)$ of all isometries of $(M, g)$ is a closed subgroup of $C(M, g)$. A subgroup $G$ of $C(M, g)$ is said to be essential if $G$ is not contained in $I\left(M, e^{2 \rho} g\right)$ for any smooth function $\rho$ on $M$, and is said to be inessential otherwise.

In this paper, unless otherwise stated, we always assume $\operatorname{dim} M>2$, although some of our propositions are valid even for $\operatorname{dim} M=2$.

There have been two conjectures:

Conjecture I. Let $(M, g)$ be a compact Riemannian n-manifold. Then $C_{0}(M, g)$ is essential if and only if $(M, g)$ is conformorphic to a Euclidean $n$ sphere $S^{n}$.

Conjecture II. Let $(M, g)$ be a compact Riemannian n-manifold with constant scalar curvature $k$. Then $C_{0}(M, g)$ is essential if and only if $k$ is positive and $(M, g)$ is isometric to a Euclidean $n$-sphere $S^{n}(k)$ of radius $1 / \sqrt{k}$. 
In each of the conjectures, "if" part is obvious.

Conjecture I has been proved under some additional conditions, for example, in the following cases:

(a) $C_{0}(M, g)$ contains a one-parameter subgroup generated by a gradient vector field (Ishihara-Tashiro [11], Tashiro [33]).

(b) $C_{0}(M, g)$ is transitive on $M$ (Nagano [21], Ba [2]).

(c) $C_{0}(M, g)$ contains a one-parameter subgroup generated by a vector field with singular points at each of which its divergence does not vanish (Avez [1], Obata [26]).

(d) $(M, g)$ is conformally flat and has a finite fundamental group (Obata [27]).

(e) $(M, g)$ is analytic and has a finite fundamental group (Ledger-Obata [16]).

Recently Lelong-Ferrand [17] has proved Conjecture I by using a technique involving quasi-conformal transformations. In the present paper Conjecture I will be proved along with the proof in [16]; indeed, the assumptions of analyticity and finite fundamental group will be removed. Our method is different from Lelong-Ferrand's in idea.

As for Conjecture II there have been also many results under some additional conditions, for example, in the following cases:

(f) $(M, g)$ is an Einstein space (Yano-Nagano [31]).

(g) $(M, g)$ is a Riemannian manifold with parallel Ricci tensor (Tanaka [31], Nagano [22]).

(h) $(M, g)$ is homogeneous (Goldberg-Kobayashi [6], [7]).

(i) The magnitude of the Ricci tensor or the curvature tensor is constant (Lichnerowicz [19], Barbance [3], Hsiung [9]).

(j) $C_{0}(M, g)$ contains a one-parameter subgroup generated by a gradient vector field (Ishihara-Tashiro [11], Obata [24], [25], Lichnerowicz [19], Tashiro [33], Yano-Obata [36], Bishop-Goldberg [4], Tanno-Weber [32]).

It should be remarked that in some of the above results, compactness is replaced by a weaker condition of completeness, and an essential conformal vector field is replaced by a nonisometric conformal vector field. In most of the above cases the additional conditions are made to reduce the problem to the case of a conformal vector field which is a gradient of some function; this situation is typical in an Einstein space. A gradient conformal vector field is essential in our terminology.

Once Conjecture $\mathrm{I}$ is proved, the manifold under consideration in Conjecture II may be thought of as an $n$-sphere with a Riemannian metric which is conformal to the standard one and has constant scalar curvature. Thus Conjecture II follows from a result on a conformal change of metrics, namely, in the above case such a conformal metric is of constant (sectional) curvature (Proposition 6.1). 
This paper is divided into two parts, one for Conjecture I and the other for Conjecture II.

$\S 1$ contains some preliminary facts about the conformal structure on a Riemannian manifold. In particular, by following the theory of $G$-structures the topology of the group $C(M, g)$ of all conformorphisms will be given. Proofs are omitted mostly because they are just translations of known results in the general theory.

$\S 2$ contains several propositions on essential groups of conformorphisms, each of which will be used later. Whenever the references are known, the proofs are omitted.

In $\S 3$, known results on conformorphisms of a Euclidean sphere will be quoted mainly from [26], and an improvement of a theorem in [27] will be given and proved.

In $\S 4$, after showing that if $C_{0}(M, g)$ is essential then $(M, g)$ is conformally flat, we shall prove Conjecture I by using the same idea as that given in [16].

In Part II, after preparing general formulas for conformal changes of metric in $\S 5$, a special case for a Euclidean sphere will be considered in $\S 6$, which seems to be a clue for the solution of Conjecture II.

In Part I and consequently in Part II as well, the following theorem of Kuiper [14] will be of essential use, in particular, in the proof of Proposition 3.4.

Theorem K. A conformally flat simply connected Riemannian n-manifold is conformorphic to an open submanifold of a Euclidean n-sphere.

An outline of this paper has been announced in [28].

\section{PART I}

\section{Preliminary remarks}

Let $(M, g)$, or simply $M$, be a Riemannian $n$-manifold with Riemannian metric $g$. From the general theory of $G$-structures of finite type (Kobayashi [12], [13], Sternberg [30]) it follows that the group $C(M, g)$ of all conformorphisms of $(M, g)$ is a Lie group with respect to the compact-open topology, since a conformal structure is indeed of finite type for $n>2$. We shall give some necessary facts in our terminology. A conformal frame at a point $p$ of $M$ is, by definition, a triple $b=\left(\lambda, b_{0}, \mu\right)$, where $\lambda$ is a positive number, $b_{0}$ an orthonormal frame with respect to the Riemannian structure on $M$, and $\mu$ a nonzero tangent vector at $p$ (Cartan [5]). The set $P$ of all the conformal frames of $M$ is equivalent to a sub-bundle of the bundle $P^{2}(M)$ of 2-jects of $M$ (Ogiue [29]). The bundle $P$ is called the conformal frame bundle of $M$, which is known to be completely parallelizable, i.e., to enjoy an $\{e\}$-structure. A conformorphism 
of $(M, g)$ is identified with a fibre preserving diffeomorphism of $P^{2}(M)$ leaving $P$ invariant, i.e., with an automorphism of the $\{e\}$-structure of $P$ by a prolongation. By a theorem of Kobayashi (Kobayashi [12], [13]; Sternberg [30]) on automorphisms of a manifold with complete parallelism, we can state the following propositions.

Proposition 1.1. $C(M, g)$ acts on the conformal frame bundle $P$ without fixed points.

Proposition 1.2. Let $\left\{f_{k}\right\}$ be a sequence of elements of $C(M, g)$ such that $f_{k}(b) \rightarrow f(b)$ for some conformal frame $b$ on $M$ and some $f \in C(M, g)$. Then $f_{k} \rightarrow f$ in the topology of $C(M, g)$.

Proposition 1.3. Let $\left\{f_{k}\right\}$ be a sequence of elements of $C(M, g)$ such that $f_{k}(b) \rightarrow b^{\prime}$ for some conformal frames $b$ and $b^{\prime}$ on $M$. Then there exists an $f \in C(M, g)$ such that $f(b)=b^{\prime}$.

As an easy consequence of these propositions the following is obtained.

Proposition 1.4. Let $M^{\prime}$ be an open submanifold of $M$, which is invariant under the action of $C(M, g)$. Then $C(M, g)$ acts on $\left(M^{\prime}, g^{\prime}\right)$ effectively as a closed subgroup of $\left(M^{\prime}, g^{\prime}\right)$, where $g^{\prime}$ is the restriction of $g$ to $M^{\prime}$.

To close this section, for later use we give a condition for a group of isometries to be compact.

Proposition 1.5 (Ledger-Obata [16]). A closed subgroup $G$ of $I(M, g)$ is compact if and only if there exists a point $p \in M$ such that the orbit $G(p)$ through $p$ is compact.

\section{Essential groups of conformorphisms}

A subgroup $G$ of $C(M, g)$ is said to be essential if $G$ is not contained in $I\left(M, e^{2 \rho} g\right)$ for any smooth function $\rho$.

Proposition 2.1 (Ishihara [10]). An essential group of conformorphisms is not compact.

Since the group of isometries of any compact Riemannian manifold is compact, the converse is true for a compact Riemannian manifold. More precisely, we can state in the following way.

Proposition 2.2. Let $(M, g)$ be a compact Riemannian manifold. Then a closed subgroup $G$ of $C(M, g)$ is essential if and only if it is not compact.

By a theorem of Montgromery and Zippin [20], any noncompact Lie group of positive dimensions contains a closed one-parameter subgroup isomorphic to the additive group of reals. Therefore by Proposition 2.2 we obtain

Proposition 2.3. If $C_{0}(M, g)$ is essential on a compact Riemannian manifold $M$, then it contains a closed essential one-parameter subgroup.

The conformal curvature tensor $W$ of type $(1,3)$ on $(M, g)$ is a conformal invariant and therefore is invariant under the action of $C(M, g)$. It is well-known that if $W$ vanishes identically and $\operatorname{dim} M>3$, then $(M, g)$ is conformally flat. 
If $\operatorname{dim} M=3$, then $W$ automatically vanishes and it is known that there is a tensor field $\tilde{W}$ of type $(0,3)$ constructed from the Riemannian structure $g$ such that $(M, g)$ is conformally flat if and only if $\tilde{W}$ vanishes identically. $\tilde{W}$ is a conformal invariant and is invariant by the action of $C(M, g)$ as well in case $\operatorname{dim} M=3$ (see, for example, Yano [34]).

Proposition 2.4 (Hlavatý [8], Nagano [21]). If $(M, g)$ has non-vanishing conformal curvature tensor, then $C(M, g)$ is inessential.

In fact, $\|W\| g$ is invariant under the action of $C(M, g)$, and so is $\|\tilde{W}\|^{2 / 3} g$ for $\operatorname{dim} M=3$, where $\|W\|$ denotes the magnitude of $W$ with respect to $g$.

Let $G$ be a one-parameter group of conformorphisms, and $X$ the vector field defined by $G$, which is called a conformal vector field. $X$ is obviously invariant under the action of $G$ itself. A fixed point of $G$ is a zero, or a singular point, of $X$.

Proposition 2.5 (Avez [1], Obata [26]). An essential one-parameter group of conformorphisms always has a fixed point.

In fact, if $G$ has no fixed point, then $X$ never vanishes. Since $X$ is invariant under the action of $G$, so is $\bar{g}=g /\|X\|^{2}$, which is conformal to $g$. Thus $G$ is a subgroup of $I(M, \bar{g})$.

Proposition 2.6. Let $(M, g)$ be a Riemannian manifold, $(\bar{M}, \bar{g})$ a Riemannian covering manifold, and $\pi: \bar{M} \rightarrow M$ the projection with $\pi^{*} g=\bar{g}$.

(i) Then $C_{0}(M, g)$ acts on $\bar{M}$ as a closed subgroup of $C_{0}(\grave{M}, \vec{g})$.

(ii) If a closed one-parameter subgroup $G$ is essential on $M$, then so is it on $\bar{M}$.

Proof. (i) By the covering homotopy theorem any one-parameter subgroup of $C_{0}(M, g)$ acts on $\bar{M}$. Thus we have a map $\alpha: C_{0}(M, g) \rightarrow C_{0}(\bar{M}, \bar{g})$ with $\pi \circ \alpha(f)=f \circ \pi$ for all $f \in C_{0}(M, g)$. By Proposition 1.1, $\alpha$ is injective. By Propositions 1.2 and 1.3 it is easy to see that a sequence $\left\{f_{k}\right\}$ converges in $C_{0}(M, g)$ if and only if $\left\{\alpha\left(f_{k}\right)\right\}$ converges in $C_{0}(\bar{M}, \bar{g})$. Thus $\alpha$ is continuous and closed.

(ii) Let $G$ be a closed one-parameter subgroup of $C(M, g)$, which is essential on $M$. To show that $G$ is essential on $\bar{M}$, assume the contrary, where we write simply $G$ instead of $\alpha(G)$. Let $\bar{h}$ be a Riemannian metric on $\bar{M}$ conformal to $\bar{g}$ such that $G \subset I_{0}(\bar{M}, \bar{h})$. By Proposition 2.5, $G$ has a fixed point $p$ on $M$, and thus any point $\tilde{p}$ of $\bar{M}$ covering $p$ is a fixed point of $G$ on $\bar{M}$. Therefore $G$ is contained in the isotropy subgroup of $I(\bar{M}, \bar{h})$ at $\tilde{p}$, which is compact. Since $G$ is closed, it is compact, contrary to our assumption that $G$ is non-compact. Hence the proposition is proved.

Next, we consider a sufficient condition for a one-parameter group $G$ of conformorphisms to be essential. Let $X$ be the conformal vector field defined by $G$, and assume that $G$ has fixed points. It is known that the values of the divergence $\phi$ of $X$ at the fixed points are unchanged by any conformal change of metric, even though $\phi$ itself changes as a scalar function. So, if $G$ is in- 
essential, then the divergence must vanish at each of the fixed points of $G$, because any Killing vector field has vanishing divergence. Hence we have

Proposition 2.7 (Obata [26]). If a conformal vector field has non-vanishing divergence at one of its singular points, then it is essential.

However, it should be remarked that on $S^{n}$ there exists an essential conformal vector field with vanishing divergence at each of its singular points.

The following has been proved.

Proposition 2.8 (Avez [1], Obata [26]). If a Riemannian manifold $M$ admits a one-parameter group of conformorphisms with fixed points at each of which the divergence of the corresponding vector field does not vanish, then $M$ is conformorphic to a Euclidean n-sphere $S^{n}$ or a once-punctured n-sphere $S^{n}-\left\{p_{\infty}\right\}$.

\section{Conformorphisms of $S^{n}$}

As a model of Riemannian manifold admitting an essential group of conformorphisms, we consider a Euclidean $n$-sphere $S^{n}$ with standard metric and list some known facts for later use.

Proposition 3.1 (Ledger-Obata [16]). A local one-parameter group of local conformorphisms of $S^{n}$ can be extended uniquely to a global one-parameter group of global conformorphisms.

This is based on a fact that $S^{n}$ is analytic and simply connected.

A classification of essential one-parameter groups of conformorphisins is made by the following.

Proposition 3.2 (Obata [26]). Let $G=\left\{f_{t}\right\}$ be an essential one-parameter group of conformorphisms of $S^{n}$, and $X$ the vector field defined by $G$. Then $G$ has one of the following properties.

(i) $G$ has exactly one fixed point $p_{0}$ at which the divergence of $X$ vanishes, and the orbit $G(p)$, for any $p \in S^{n}$, satisfies

$$
\lim _{t \rightarrow \pm \infty} f_{t}(p)=p_{0} .
$$

(ii) $G$ has exactly two fixed points $p_{0}$ and $p_{\infty}$ at each of which the divergence of $X$ does not vanish and the orbit $G(p)$, for $p \in S^{n}-\left\{p_{0}, p_{\infty}\right\}$, connects $p_{0}$ and $p_{\infty}$.

Proposition 3.3. Let $M$ be an open submanifold of $S^{n}$, which is invariant by a one-parameter group $G$ of conformorphisms of $S^{n}$. If $G$ is essential on $M$, then $M$ is either $S^{n}$ itself or $S^{n}-\left\{p_{\infty}\right\}$.

Proof. Since $G$ is essential on $M$, so is it on $S^{n}$. Then Proposition 3.2 implies that $G$ has at most two fixed points on $S^{n}$. On the other hand, $G$ has at least one fixed point on $M$ by Proposition 2.5. Thus by Proposition 3.2, $M$ is either $S^{n}$ or $S^{n}-\left\{p_{\infty}\right\}$.

Proposition 3.4. Let $(M, g)$ be a conformally flat Riemannian n-manifold. 
It there is a closed essential one-parameter subgroup $G$ of $C_{0}(M, g)$, then $M$ is conformorphic to either $S^{n}$ or $S^{n}-\left\{p_{\infty}\right\}$.

Proof. Take the universal Riemannian covering manifold $(\bar{M}, \bar{g})$ of $(M, g)$. Then $(\grave{M}, \bar{g})$ is a simply connected conformally flat Riemannian manifold, and is therefore conformorphic to an open submanifold $N$ of $S^{n}$ by Theorem K (Kuiper [14]). Since $G$ is closed in $C_{0}(M, g)$ and essential on $M$, so is it on $\vec{M}$ by Proposition 2.6. By the conformorphism between $\bar{M}$ and $N, G$ acts on $N$, and by Proposition 3.1 the action is extended to $S^{n}$. Then by Proposition 3.3, $N$ is $S^{n}$ itself or $S^{n}-\left\{p_{\infty}\right\}$. Thus $(\bar{M}, \bar{g})$ is conformorphic to either $S^{n}$ or $S^{n}-\left\{p_{\infty}\right\}$.

The fixed points of $G$ on $\bar{M}$ are exactly the points of $\bar{M}$ covering the fixed points of $G$ on $M$. Since $G$ has at most two fixed points on $\bar{M}, \tilde{M}$ is $M$ itself or a double covering of $M$. We are going to show that $M$ itself is simply connected.

If $\bar{M}$ is a double covering of $M$, then $G$ must have two fixed points on $\bar{M}$, both of which cover a single fixed point of $G$ on $M$. Then by Proposition 3.2 the corresponding vector field $\bar{X}$ on $\bar{M}$ has nonvanishing divergence at each of these fixed points on $M$, and so does the corresponding vector field $X$ on $M$. Thus by Proposition 2.8, M itself is conformorphic to $S^{n}$ or $S^{n}-\left\{p_{\infty}\right\}$, each of which is simply connected, a contradiction.

Proposition 3.5. Let $(M, g)$ be a compact conformally flat Riemanniann $n$-manifold. If $C_{0}(M, g)$ is essential, then $M$ is conformorphic to $S^{n}$.

Proof. Since $M$ is compact, and $C_{0}(M, g)$ is essential, by Proposition 2.3 there is a closed essential one-parameter subgroup. Then by Proposition 3.4, $M$ is conformorphic to $S^{n}$ or $S^{n}-\left\{p_{\infty}\right\}$. Since $M$ is compact, it is conformorphic to $S^{n}$.

\section{Conjecture I}

Theorem I. Conjecture I is a true.

On account of Proposition 3.5 we have only to show that $(M, g)$ under consideration is conformally flat. Thus the following Proposition 4.1 together with Proposition 3.5 gives the proof of Theorem 1 .

Proposition 4.1. Let $(M, g)$ be a compact Riemannian manifold with the essential group $C_{0}(M, g)$ of conformorphisms. Then $(M, g)$ is conformally flat.

Proof. Assume that $(M, g)$ is not conformally flat, and let $N=\left\{p \in M: W_{p}\right.$ $\neq 0\}$. In case $\operatorname{dim} M=3, N=\left\{p \in M: \tilde{W}_{p} \neq 0\right\}$. Then $N$ is an open subset of $M$, and any connected component $N_{0}$ of $N$ is an open submanifold of $M$. Since $W$, as well as $\tilde{W}$ for $\operatorname{dim} M=3$, is invariant under the action of $C(M, g)$, it follows that $N$ is fixed under this action. Hence $N_{0}$ is fixed under the action of $C_{0}(M, g)$. Let $g_{0}$ be the restriction of $g$ to $N_{0}$. Then by Proposition 1.4, $C_{0}(M, g)$ acts on $N_{0}$ effectively as a closed subgroup of $C_{0}\left(N_{0}, g_{0}\right)$, which is identical with the group $I_{0}\left(N_{0}, \bar{g}_{0}\right)$ of isometrics for some $\bar{g}_{0}$ conformal to $g_{0}$ by 
Proposition 2.4. Since $C_{0}(M, g)$ is essential, by Proposition 2.3 it contains a closed essential one-parameter subgroup $G$. Then $G$ is closed in $I_{0}\left(N_{0}, \bar{g}_{0}\right)$, and hence the orbit $G(p)$, for $p \in N_{0}$, is a closed submanifold of $N_{0}$. Since $G$ is closed in $I_{0}\left(N_{0}, \bar{g}_{0}\right)$ and noncompact, it follows from Proposition 1.5 that $G(p)$ is noncompact for any $p \in N_{0}$ and is diffeomorphic to $G$ itself by the natural projection $G \rightarrow G(p)$.

Let $X$ be the conformal vector field on $(M, g)$ defined by $G$. Then $X$ is nowhere zero in $N_{0}$, since $G(p)$ is diffeomorphic to $G$.

Now on $M$ we put

$$
\begin{gathered}
F(p)=\|X \otimes X \otimes W\|_{p} \\
(F(p)=\|X \otimes X \otimes X \otimes \tilde{W}\| \text { if } \operatorname{dim} M=3),
\end{gathered}
$$

where we write $\|T\|$ for the magnitude of a tensor $T$ with respect to $g$.

Since $X$ and $W$ (or $\tilde{W}$ if $\operatorname{dim} M=3$ ) are invariant under the action of $G$, so is $X \otimes X \otimes W$ (or $X \otimes X \otimes X \otimes \tilde{W}$ if $\operatorname{dim} M=3$ ). As $F$ is of type $(3,3)$, its magnitude is invariant by $G$ as well. Thus $F$ is a nonzero constant on $G(p)$, $p \in N_{0}$. Now take $q \in \mathrm{Cl} G(p), p \in N_{0}$, the closure of $G(p)$ in $M$. Then, by the continuity of $F, F$ is a nonzero constant on $\mathrm{Cl} G(p)$ so that $F(q) \neq 0$. Thus $W_{q} \neq 0$ (or $\tilde{W}_{q} \neq 0$ if $\operatorname{dim} M=3$ ) and $q \in N$. Since $G(p)$ is closed in $N_{0}$, we have $q \in G(p)$. Thus $G(p)$ is closed in the compact manifold $M$ and hence is a compact submanifold of $M$ and $N_{0}$ as well. Since $G$ is a closed subgroup of $I_{0}\left(N_{0}, \bar{g}_{0}\right)$, it follows from Proposition 1.5 that $G$ is compact and so we have a contradiction. Thus $W$ (and $\tilde{W}$ if $\operatorname{dim} M=3$ ) must vanish identically and $M$ must be conformally flat.

Remark. A Euclidean $n$-sphere has the essential group of conformorphisms, and so the "if" part of the conjecture is obvious.

\section{PART II}

\section{General formulas for conformal changes of metric}

Let $M$ be a Riemannian $n$-manifold. With respect to a local coordinate system we use $g_{i j},\left\{\begin{array}{l}h \\ j i\end{array}\right\}, \nabla_{i}, K_{k j i}{ }^{h}, K_{j i}=K_{h j i}{ }^{h}, K=K_{j i} g^{j i}$, and $k=$ $K /[n(n-1)]$, to denote, respectively, the metric tensor, the Christoffel symbols formed with $g_{j i}$, the operator of covariant differentiation with respect to $\left\{\begin{array}{l}h \\ j i\end{array}\right\}$, the curvature tensor, the Ricci tensor, the contracted curvature scalar and the scalar curvature of $M$. Put

$$
G_{j i}=K_{j i}-K g_{j i} / n
$$


which measures the deviation of $M$ from being an Einstein space.

Consider a conformal change of metric

$$
g_{j i}^{*}=e^{2 \rho} g_{j i} .
$$

When $\Omega$ is a quantity formed with $g$, we denote by $\Omega^{*}$ the corresponding quantity formed with $g^{*}$. For later convenience, we put

$$
u=e^{-\rho}, \quad u_{i}=\nabla_{i} u .
$$

Then the following formulas are known (Yano-Obata [37]):

$$
\begin{gathered}
K^{*}=u^{2} K+2(n-1) u \Delta u-n(n-1) u_{i} u^{i}, \\
G_{j i}^{*}=G_{j i}+(n-2) P_{j i},
\end{gathered}
$$

where

$$
\begin{gathered}
\Delta u=g^{j i} \nabla_{j} u_{i}, \\
P_{j i}=u^{-1}\left(\nabla_{j} u_{i}-\Delta u g_{j i} / n\right), \quad P_{j}^{i}=P_{j k} g^{k i} .
\end{gathered}
$$

From (5.7) we obtain

$$
P_{j i} P^{j i}=u^{-2}\left[\nabla^{j} u^{i} \nabla_{j} u_{i}-(\Delta u)^{2} / n\right] .
$$

\section{Conformal changes of metrics on $S^{n}$}

On a Riemannian manifold one can consider a conformal change corresponding to an arbitrarily given function $\rho$ in (5.2), However, if there is given a curvature condition for the changed metric, then in general the existence of a conformal change satisfying the condition is not known. We are going to prove the following proposition, which is a clue for the solution of Conjecture II.

Proposition 6.1. Let $\left(S^{n}, g\right)$ be a Euclidean $n$-sphere of radius 1 , and $g^{*}$ another Riemannian metric on $S^{n}$ conformal to $g$. Then $g^{*}$ is of constant scalar curvature 1 if and only if it is of constant (sectional) curvature 1.

Proof. We have $G_{j i}=0$ and $K=n(n-1)$ on $\left(S^{n}, g\right)$, and $K^{*}=n(n-1)$ on $\left(S^{n}, g^{*}\right)$. Therefore from (5.4) and (5.5) it follows that

$$
\begin{aligned}
\Delta u & =\frac{n}{2} u^{-1}\left(1-u^{2}+u_{i} u^{i}\right), \\
G_{j i}^{*} & =(n-2) P_{j i} .
\end{aligned}
$$

By using (6.1) and (6.2) we shall show that $P_{j i}$ and therefore $G_{j i}^{*}$ vanish identically. To do this, consider a nonnegative quantity

$$
A=u^{3-n} P_{j i} P^{j i}=u^{1-n}\left[\nabla_{j} u_{i} \nabla^{j} u^{i}-(\Delta u)^{2} / n\right],
$$


and a vector field

$$
v^{i}=u^{2-n} u^{j} P_{j}^{i}=u^{1-n}\left[u^{j} \nabla_{j} u^{i}-(\Delta u) u^{i} / n\right] .
$$

A straightforward computation then gives

$$
\nabla_{i} v^{i}=A+B
$$

where

$$
\begin{aligned}
B= & (1-n) u^{-n} u^{j} u^{i} \nabla_{j} u_{i}+\frac{n-1}{n} u^{-n}\left[u u^{j} \nabla_{j}(\Delta u)+u_{i} u^{i} \Delta u\right] \\
& +u^{1-n} u^{j}\left(\nabla_{i} \nabla_{j} u^{i}-\nabla_{j} \nabla_{i} u^{i}\right) .
\end{aligned}
$$

Substituting (6.1) in the second term and applying the Ricci formula to the last term on the right hand side of (6.3), we get

$$
\begin{aligned}
& \text { the second term }=(n-1) u^{-n}\left(u^{j} u^{i} \nabla_{j} u_{i}-u u_{i} u^{i}\right), \\
& \text { the last term }=(n-1) u^{1-n} u_{i} u^{i} .
\end{aligned}
$$

Thus $B=0$, and therefore $\nabla_{i} v^{i}=A \geq 0$. By the well-known Bochner's lemma we obtain that $A=0$, so that $P_{j i}=0$ and therefore

$$
G_{j i}^{*}=0,
$$

which implies that $\left(S^{n}, g^{*}\right)$ is an Einstein space. Since $g^{*}$ is conformal to $g$, $\left(S^{n}, g^{*}\right)$ is conformally flat. It is known that a conformally flat Einstein space is always a space of constant (sectional) curvature. Hence $g^{*}$ has constant (sectional) curvature 1 .

Remark 1. A little more general argument, similar to this proof, may be seen in Yano-Obata [37, Proposition 3.3].

Remark 2, In the proof of Proposition 6.1, one can see that $G_{j i}=0$ implies $G_{j i}^{*}=(n-2) P_{j i}=0$ under the condition $k=k^{*}=1$. Since $P_{j i}=0$ implies that the manifold under consideration is isometric to a unit sphere (Lichnerowicz [19], Yano-Obata [36]), we obtain

Proposition 6.2. Let $(M, g)$ be a compact Einstein space with scalar curvature 1. If there is a Riemannian metric $g^{*}(\neq g)$ on $M$ such that $g^{*}$ is conformal to $g$ and $g^{*}$ has a constant scalar curvature 1 , then $(M, g)$ and $\left(M, g^{*}\right)$ are isometric to a unit n-sphere.

Remark 3. $\left(S^{n}, g^{*}\right)$ can be obtained by a conformorphism of $\left(S^{n}, g\right)$. The proof of this will be given in a forthcoming paper.

\section{Cojecture II}

Theorem II. Conjecture II is true.

Proof. It is known (Kurita [15], Lichnerowicz [18], Obata [23]) that if a compact Riemannian manifold with constant scalar curvature admits a non- 
isometric conformorphism, then the constant scalar curvature is positive. Therefore without loss of generality we may assume that the Riemannian manifold $(M, g)$ under consideration has constant scalar curvature 1 . Since $C_{0}(M, g)$ is essential, it follows from Theorem I that there exists a conformorphism $f$ of $(M, g)$ onto $\left(S^{n}, g_{0}\right)$ where $g_{0}$ is a standard metric on a unit sphere $S^{n}$. Thus $\left(f^{-1}\right)^{*} g=g^{*}$ is a Riemannian metric on $S^{n}$ and $f:(M, g) \rightarrow\left(S^{n}, g^{*}\right)$ is an isometry. Since $g^{*}$ is conformal to $g_{0}$ and $g^{*}$ has scalar curvature 1 , it follows from Proposition 6.1 that $\left(S^{n}, g^{*}\right)$ is of constant (sectional) curvature. Thus $(M, g)$ is isometric to a Euclidean $n$-sphere $\left(S^{n}, g^{*}\right)$.

Remark. It is not difficult to show that the one-parameter subgroup of $C_{0}(M, g)$ generated by the gradient of a certain function on $M$ is a closed essential subgroup of $C_{0}(M, g)$.

\section{Bibliography}

[1] A. Avez, Transformations conformes des variétés riemanniennes compactes, C. R. Acad. Sci. Paris 259 (1964) 4469-4472; 260 (1965) 1550-1553.

[2] B. Ba, Structure presque complexe, structure conforme et dérivations, Cahiers de Topologie et Géométrie Différentielle, C.N.R.S. Paris 8 (1966).

[ 3 ] C. Barbance, Transformations conformes d'une variété riemannienne compacte, C. R. Acad. Sci. Paris 260 (1965) 1547-1549.

[4] R. L. Bishop \& S. I. Goldberg, A characterization of the Euclidean sphere, Bull. Amer. Math. Soc. 72 (1966) 122-124.

[ 5 ] E. Cartan, Les espaces à connexion conforme, Ann. Soc. Polon. Math. 2 (1923) 171-221.

[6] S. I. Goldberg \& S. Kabayashi, The conformal transformation group of a compact homogeneous manifold, Bull. Amer. Math. Soc. 68 (1962) 378-381.

[7] _ The conformal transformation group of a compact Riemannian manifold, Amer. J. Math. 84 (1962) 170-174.

[ 8 ] V. Hlavatý, Zur Konformgeometrie, Proc. Acad. Amsterdam 38 (1935) 281-286.

[9] C. C. Hsiung, On the group of conformal transformations of a compact Riemannian manifold. I, II, III, Proc. Nat. Acad. Sci. U.S.A. 54 (1965) 1509-1513; Duke Math. J. 34 (1967) 337-341; J. Differential Geometry 2 (1968) 185-190.

[10] S. Ishihara, Groups of projective transformations and groups of conformal transformations, J. Math. Soc. Japan 9 (1957) 195-227.

[11] S. Ishihara \& Y. Tashiro, On Riemannian manifolds admitting a concircular transformation, Math. J. Okayama Univ. 9 (1959) 19-47.

[12] S. Kabayashi, Le groupe de transformations qui laissent invariant un parallélisme, Colloque de Topologie, Ehresmann, Strasbourge, 1954.

[13] _- Theory of connections, Ann. Mat. Pura Appl. 43 (1957) 119-194.

[14] N. H. Kuiper, On conformally-flat spaces in the large, Ann. of Math. 50 (1949) 916-924.

[15] M. Kurita, A note on conformal mappings of certain Riemannian manifolds, Nagoya Math. J. 21 (1962) 111-114.

[16] A. J. Ledger \& M. Obata, Compact riemannian manifolds with essential groups of conformorphisms, Trans. Amer. Math. Soc. 150 (1970) 645-651.

[17] J. Lelong-Ferrand, Transformations conformes et quasiconformes des variétés riemanniennes; application à la démonstration d'une conjeceure de A. Lichnerowicz, C. R. Acad. Sci. Paris 269 (1969) 583-586.

[18] A. Lichnerowicz, Géométrie des groupes de transformations, Paris, 1958.

[19] - Sur les transformations conformes d'une variété riemannienne compacte, C. R. Acad. Sci. Paris 259 (1964) 697-700. 
[20] D. Montgomery \& L. Zippin, Existence of subgroups isomorphic to the real numbers, Ann. of Math. 53 (1951) 298-326.

[21] T. Nagano, On conformal transformations of Riemannian spaces, J. Math. Soc. Japan 10 (1958) 79-93.

[22] _ The conformal transformations on a space with parallel Ricci curvature, J. Math. Soc. Japan 11 (1959) 10-14.

[23] M. Obata, Conformal transformations of compact Riemannian manifolds, Illinois J. Math. 6 (1962) 292-295.

[24] _- Certain conditions for a Riemannian manifold to be isometric with a sphere, J. Math. Soc. Japan 14 (1962) 333-340.

[25] _- Riemannian manifolds admitting a solution of a certain system of differential equations, Proc. U.S.-Japan Sem. Differential Geometry, Kyoto, Japan, 1965, 101-114.

[26] —- Conformal transformations of Riemannian manifolds, J. Differential Geometry 4 (1970) 311-333.

[27] - Conformally flat Riemannian manifolds admitting a one-parameter group of conformal transformations, J. Differential Geometry 4 (1970) 335-337.

[28] _- The conjectures on conformal transformations of Riemannian manifolds, Bull. Amer. Math. Soc. 77 (1971) 265-270.

[29] K. Ogiue, Theory of conformal connections, Kōdai Math. Sem. Rep. 19 (1967) 193-224.

[30] S. Sternberg, Lectures on differential geometry, Prentice-Hall, Englewood Cliffs, N. J., 1964.

[31] N. Tanaka, Conformal connections and conformal transformations, Trans. Amer. Math. Soc. 92 (1959) 168-190.

[32] S. Tanno \& W. C. Weber, Closed conformal vector fields, J. Differential Geometry 3 (1969) 361-366.

[33] Y. Tashiro, Complete Riemannian manifolds and some vector fields, Trans. Amer. Math. Soc. 117 (1965) 251-275.

[34] K. Yano, The theory of Lie derivatives and its applications, North-Holland, Amsterdam, 1957.

[35] K. Yano \& T. Nagano, Einstein spaces admitting a one-parameter group of conformal transformations, Ann. of Math. 69 (1959) 451-461.

[36] K. Yano \& M. Obata, Sur le groupe de transformations conformes d'une variété de Riemann dont le scalaire de courbure est constant, C. R. Acad. Sci. Paris 260 (1965) 2698-2700.

[37] —_, Conformal changes of Riemannian metrics, J. Differential Geometry 4 (1970) 53-92.

Tokyo Metropolitan University UNIVERSITY OF DURhaM 